\title{
Construction of a recombinant pIRES2-EGFP-ARTS plasmid and its effect on LX-2 cells
}

\author{
FEIFAN XU ${ }^{1 *}$, YUANLONG HAN $^{1 *}$, DANDAN ZHU ${ }^{2}$, HUA TIAN ${ }^{1}$, \\ HUIMING ZHU ${ }^{1}$, JINGJING REN ${ }^{1}$, DELIN GU ${ }^{1}$ and YINONG DUAN ${ }^{2}$ \\ ${ }^{1}$ Clinical Laboratory, The Sixth People's Hospital of Nantong, Nantong, Jiangsu 226011; ${ }^{2}$ Department of Pathogen Biology, \\ School of Medicine, Nantong University, Nantong, Jiangsu 226009, P.R. China
}

Received October 28, 2016; Accepted June 13, 2017

DOI: $10.3892 / \mathrm{mmr} .2017 .7172$

\begin{abstract}
The inhibition of the activation of hepatic stellate cells (HSCs) and the induction of their apoptosis have been investigated as potential strategies to counteract the development and progression of liver fibrosis. Previous research has suggested that apoptosis-related protein in the transforming growth factor- $\beta$ signaling pathway (ARTS) may serve a significant role in numerous cell types; however, little is known regarding its roles in HSCs. Total RNA was extracted from LX-2 cells, and the human full-length ARTS gene was obtained by reverse transcription-polymerase chain reaction and inserted into the pIRES2-EGFP cloning vector. Subsequently, the recombinant pIRES2-EGFP-ARTS plasmid was transfected into LX-2 cells by FuGENE 6 transfection reagent, and the expression of ARTS was detected by western blotting and fluorescent microscopy. In addition, the effects of pIRES2-EGFP-ARTS on the activation, apoptosis, viability and migration of LX-2 cells were assessed by western blot analysis, TUNEL staining, an MTT assay, and scratch and Transwell assays, respectively. The present results demonstrated that the pIRES2-EGFP-ARTS vector expressing human ARTS was successfully constructed, and the overexpression of ARTS contributed to enhance the apoptosis and inhibit the activation of human LX-2 HSCs. The present findings
\end{abstract}

Correspondence to: Professor Delin Gu, Clinical Laboratory, The Sixth People's Hospital of Nantong, 500 Yonghe Road, Nantong, Jiangsu 226011, P.R. China

E-mail: dlgu@163.com

Professor Yinong Duan, Department of Pathogen Biology, School of Medicine, Nantong University, 19 Qixiu Road, Nantong, Jiangsu 226009, P.R. China

E-mail: yinongduan@gmail.com

${ }^{*}$ Contributed equally

Key words: apoptosis-related protein in the transforming growth factor- $\beta$ signaling pathway, eukaryotic expression vector pIRES2-EGFP, liver fibrosis, apoptosis, activation suggested that ARTS overexpression may have potential as a novel therapeutic strategy to reverse hepatic fibrosis.

\section{Introduction}

Liver fibrosis, which characterized by abnormal deposition of extracellular matrix (ECM) may be caused by various etiological factors, and is associated with a high mortality and morbidity worldwide (1). A previous study demonstrated that the pivotal pathogenic event in liver fibrosis is the activation of hepatic stellate cells (HSCs) (2). Therefore, inhibition of activated HSCs or induction of HSCs apoptosis may be an effective strategy in the treatment of hepatic fibrosis (3). LX-2 is an immortalized human HSC line that expresses a range of human hepatic fibrosis features: As hepatic fibrosis progresses, LX-2 cells are activated, the expression of $\alpha$-smooth muscle actin ( $\alpha$-SMA) increases, whereas the expression of peroxisome proliferator-activated receptor- $\gamma$ (PPAR- $\gamma$ ) decreases. Therefore, LX-2 cells have been used as the primary cell type for liver fibrosis research (4-6).

Apoptosis-related protein in the transforming growth factor- $\beta$ (TGF- $\beta$ ) signaling pathway (ARTS), also termed septin (Sept)4_i2, is a recognized splice variant encoded by the human Sept4 gene (7). Unlike other splice variants, ARTS exists in the cytomembrane and is released into the cytoplasm following stimulation by various molecules, including TGF- $\beta$, staurosporine, Fas, arabinoside and etoposide, and subsequently exhibits a proapoptotic function by binding to the X-linked inhibitor of apoptosis protein (8). Previous studies have indicated that ARTS regulates apoptosis in various cell types, including COS-7, Hela, K562, HL-60 and ALLs (9-11). Furthermore, Iwaisako et al (12) reported that Sept4 was downregulated in mice with liver fibrosis that was induced by bile duct ligation and carbon tetrachloride, which indicated that Sept4 may have an important role in liver fibrosis. However, to the best of our knowledge, it has not previously been reported whether ARTS has a role in HSC apoptosis and activation. Therefore, the current study aimed to construct the recombinant pIRES2-enhanced green fluorescent protein (EGFP)-ARTS plasmid and determine the effect of ARTS overexpression on LX-2 cells. 


\section{Materials and methods}

Materials. LX-2 cells were obtained from Xiang Ya Central Experiment Laboratory (Hunan, China). pIRES2-EGFP vector was purchased from Clontech Laboratories, Inc. (Mountainview, CA, USA) and E. coli DH5 $\alpha$ was purchased from Takara Biotechnology Co.,Ltd.(Dalian, China). Transwell chambers were preserved in our laboratory. DNA marker was purchased from Nanjing KeyGen Biotech Co., Ltd. (Nanjing, China), BamHI and HindIII restriction enzymes, RevertAid First Strand cDNA Synthesis kit and dNTPs were purchased from Fermentas (Thermo Fisher Scientific, Inc., Waltham, MA, USA). A terminal deoxynucleotidyl transferase-mediated dUTP-biotin nick end labeling assay (TUNEL) staining kit to measure apoptosis was purchased from Roche Diagnostics (Basel, Switzerland). FuGENE6 transfection reagent was purchased from Promega Corporation (Madison, WI, USA). Dimethyl sulfoxide (DMSO) and MTT reagent was purchased from Sigma-Aldrich (Merck KGaA, Darmstadt, Germany). The pEASY-T1 vector, TransStart ${ }^{\circledR}$ FastPfu DNA Polymerase, polymerase chain reaction (PCR) Stimulant and DNA ligase were purchased from Beijing Transgen Biotech Co., Ltd. (Beijing, China), and TRIzol and DNA gel extraction kit were purchased from Sangon Biotech Co., Ltd. (Shanghai, China). Primary antibodies for caspase-3 and GAPDH were purchased from Cell Signaling Technology, Inc. (Danvers, MA, USA), primary antibodies for ARTS were purchased from ProSci, Inc. (Poway, CA, USA), primary antibodies for $\alpha$-SMA and all secondary antibodies were obtained from Santa Cruz Biotechnology, Inc. (Dallas, TX, USA).

Gene amplification, cloning and identification. Total RNA was extracted from LX-2 cells by TRIzol reagent according to the manufacturer's protocol. Extracted RNA $(1 \mu \mathrm{g})$ was used for cDNA synthesis by reverse transcription (RT) using the RevertAid First Strand cDNA synthesis kit, according to the manufacturer's protocol. The temperature protocol used was as follows: At $42^{\circ} \mathrm{C}$ for $60 \mathrm{~min}$ and at $70^{\circ} \mathrm{C}$ for $5 \mathrm{~min}$. The following primer sequences were designed based on the ARTS mRNA sequence (GenBank reference, NM_080415.2): 5'-CCCTCGAGGCCACCATGATCAAGCGTTTCCTGG AGG-3' (forward) and 5'-CGGGATCCCTAGTGGCAGCCC TGCCCCTGGTG-3' (reverse). Underlined letters indicate the restriction sites. The PCR reaction system was prepared in a total volume $50 \mu \mathrm{l}$ containing $10 \mu \mathrm{l}$ 5X TransStart FastPfu reaction buffer, $1.25 \mu 1 \mathrm{dNTPs}, 1 \mu 1$ TransStart ${ }^{\circledR}$ FastPfu DNA Polymerase, $5 \mu \mathrm{l}$ PCR Stimulant, $2 \mu \mathrm{l}$ primer mix and double distilled $\mathrm{H}_{2} \mathrm{O}$ up to $50 \mu \mathrm{l}$. The PCR program was performed at $94^{\circ} \mathrm{C}$ for $3 \mathrm{~min}$, followed by 30 cycles at $94^{\circ} \mathrm{C}$ for $30 \mathrm{sec}$, $55^{\circ} \mathrm{C}$ for $30 \mathrm{sec}$ and $72^{\circ} \mathrm{C}$ for $1 \mathrm{~min}$, and completed at $72^{\circ} \mathrm{C}$ for $10 \mathrm{~min}$. The full-length ARTS was $846 \mathrm{bp}$. PCR products were separated by $1 \%$ polyacrylamide gels and the target fragment was purified by an agarose gel extraction kit.

The purified fragment was inserted into the pEASY-T1 cloning vector and $100 \mathrm{ng}$ recombined plasmid was transformed into $E$. coli DH5 $\alpha$ competent cells and verified by two pairs of PCR primers maintained in our laboratory. The primer sequences were as follows: First pair primers: 5'-ACACCAGGT TTTGGGGATG-3' (forward) and 5'-TTCCCTTGACAGCAC CCG-3' (reverse); and the second pair primers: 5'-CTGCTG
CCTGTACTTCATCTCA-3' (forward) and 5'-TGCTCTTGG CCTGTTCCCT-3' (reverse). Subsequently, pEASY-T1-ARTS and the eukaryotic expression vector pIRES2-EGFP were digested by Hind III and $\mathrm{BamHI}$ restriction enzymes at $37^{\circ} \mathrm{C}$ for 30 min simultaneously, and ligated by DNA ligase at $16^{\circ} \mathrm{C}$ for $2 \mathrm{~h}$. The recombinant plasmid was validated by double restriction enzyme digestion, separated by $1 \%$ PAGE and sequenced by Sanger sequencing by Sangon Biotech Co., Ltd. The correct recombinant plasmid was termed pIRES2-EGFP-ARTS. The construction image is presented in Fig. 1.

Transfection of pIRES2-EGFP-ARTS into LX-2 cells. LX-2 cells were maintained in Dulbecco's modified Eagle's medium (DMEM; Gibco; Thermo Fisher Scientific, Inc.) supplemented with $10 \%$ fetal bovine serum (FBS; Hyclone; GE Healthcare Life Sciences, Logan, UT, USA), and $100 \mu \mathrm{g} / \mathrm{ml}$ streptomycin solution and $100 \mathrm{U} / \mathrm{ml}$ penicillin at $37^{\circ} \mathrm{C}$ in a humidified incubator with $5 \% \mathrm{CO}_{2}$. The experiment was divided into the following three groups: Control, vector and ARTS overexpression groups. Plasmids (3-4 $\mu \mathrm{g})$ were transfected into LX-2 cells at $37^{\circ} \mathrm{C}$ for $48 \mathrm{~h}$ using FuGENE 6 with DMEM containing $2 \% \mathrm{FBS}$, according to the manufacturer's protocol. LX-2 cells $\left(1 \times 10^{6}\right.$ cells $\left./ \mathrm{ml}\right)$ were fixed in $4 \%$ paraformaldehyde for $10 \mathrm{~min}$ at room temperature. The transfection efficiency was visualized by using an inverted fluorescence microscope (Leica Microsystems GmbH, Wetzlar, Germany) at magnification, $\mathrm{x} 200$. The protein expression of ARTS was confirmed by western blotting.

Western blot analysis. For western blot analysis, after LX-2 cells were transfected with pIRES2-EGFP-ARTS for $48 \mathrm{~h}$, culture media was removed and the cells were washed with PBS. Proteins were extracted using radioimmunoprecipitation assay lysis buffer (Beyotime Institute of Biotechnology, Haimen, China) and quantified according to the Bradford method. Equal amounts $(30 \mu \mathrm{g})$ of extracted protein samples were separated by 10 or $12 \%$ SDS-PAGE followed by electroblotting onto polyvinylidene fluoride membranes (EMD Millipore, Billerica, MA, USA). Following electrophoresis, the membranes were blocked with $5-10 \%$ non-fat milk for $2 \mathrm{~h}$ at room temperature and incubated with specific primary antibodies against ARTS (cat no. 3025; 1:1,000), caspase-3 (cat no. 9665; 1:1,000), $\alpha$-SMA (cat no. 130617; 1:200) and GAPDH (cat no. $2118 ; 1: 1,000$ ) at $4{ }^{\circ} \mathrm{C}$ overnight. Subsequently, membranes were incubated with horseradish peroxidase-conjugated secondary antibodies (cat no. sc-2004; $1: 5,000)$ for $2 \mathrm{~h}$ at a room temperature. Membranes were visualized using an enhanced chemiluminescence kit (EMD Millipore). Blots were semi-quantified by densitometry using ImageJ software version 2.0 (National Institutes of Health, Bethesda, MD, USA).

TUNEL assay. Apoptosis was detected using a TUNEL kit according to the manufacturer's protocol. Briefly, cultured cells $\left(1 \times 10^{6}\right.$ cells $\left./ \mathrm{ml}\right)$ following transfection were fixed with $4 \%$ paraformaldehyde ( $\mathrm{pH}$ 7.4) for $1 \mathrm{~h}$ at a room temperature and subsequently permeabilized with $0.1 \%$ Triton-X-100 for 2 min on ice. The cells were then incubated with the TUNEL reaction mixture for $60 \mathrm{~min}$ and counterstained with Hoechst $33258(0.5 \mu \mathrm{g} / \mathrm{ml})$ for $15 \mathrm{~min}$ at room temperature. Cells were 
A

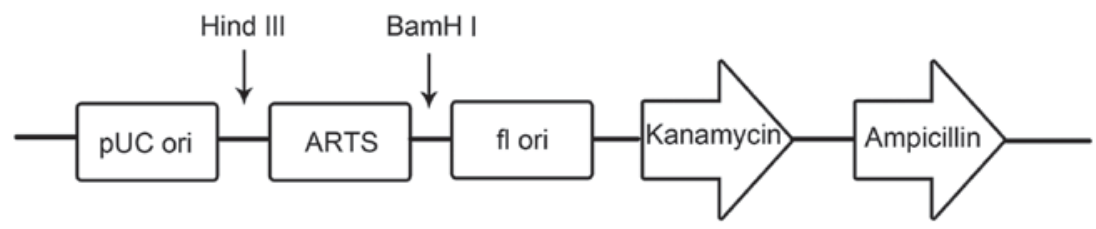

B

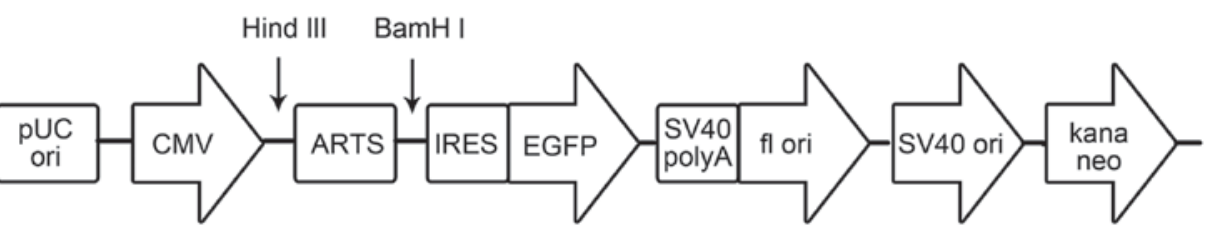

Figure 1. Schematic indicating construction of recombinant plasmids and restriction sites. The construction of the recombinant (A) pEASY-T1-ARTS and (B) pIRES2-EGFP-ARTS plasmids. ARTS, apoptosis-related protein in the transforming growth factor- $\beta$ signaling pathway; EGFP, enhanced green fluorescent protein.

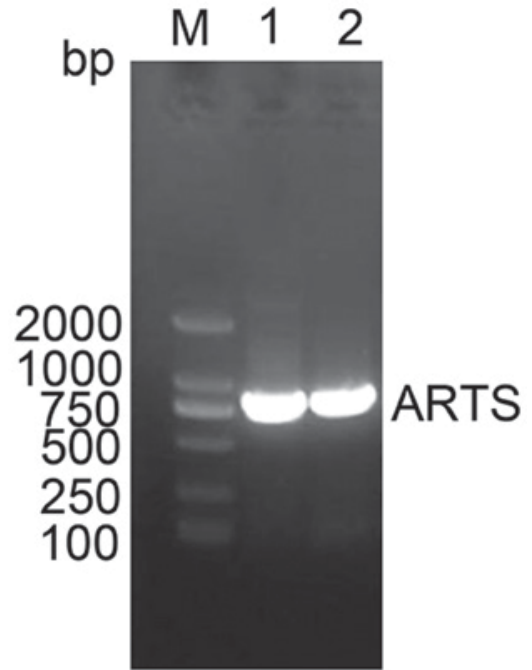

Figure 2. Amplification of ARTS gene by RT-PCR. Lane M, DL2000 DNA marker; lanes 1 and 2, RT-PCR products of ARTS gene ( $846 \mathrm{bp})$. ARTS, apoptosis-related protein in the transforming growth factor- $\beta$ signaling pathway; RT-PCR, reverse transcription-polymerase chain reaction.

washed with 1X PBS. Apoptotic cells were visualized by fluorescence microscopy in at least 10 random fields of view per cover slip.

MTT assay. Cell survival and proliferation were detected by an MTT assay. Briefly, after transfection for $48 \mathrm{~h}$, LX-2 cells were plated in a 96 -well culture plate $\left(5 \times 10^{3}\right.$ cells/well $)$ and were incubated with MTT reagent $(0.5 \mathrm{mg} / \mathrm{ml})$ for $4 \mathrm{~h}$ at room temperature. Dimethyl sulfoxide $(0.1 \%)$ was subsequently added to dissolve the formazan products for $10 \mathrm{~min}$ at room temperature. An ELISA reader was used to determine the optical density at $490 \mathrm{~nm}$ wavelength.

Scratch assay. A scratch-wound test was performed to evaluate the effect of ARTS on LX-2 migration. LX-2 cells were seeded in 6-well plates at a density of $1 \times 10^{6}$ cells $/ \mathrm{ml}$ and transfected for $48 \mathrm{~h}$, as described above. A $200 \mu \mathrm{l}$ pipette tip was used to scrape and generate a scratch wound. Following $24 \mathrm{~h}$, cell debris was removed by washing with $1 \mathrm{X}$ PBS. The migrated cells were counted with an inverted microscope under magnification, x50 to evaluate the migrated proportion in at least 5 random fields of view per well.

Transwell assay. For the Transwell migration assay, cells were resuspended at a density of $1 \times 10^{6}$ cells $/ \mathrm{ml}$ following transfection for $48 \mathrm{~h}$. Subsequently, $200 \mu \mathrm{l}$ cell suspension $\left(2 \times 10^{5}\right.$ cells $\left./ \mathrm{ml}\right)$ in DMEM was added into the upper chambers of the Transwell inserts, whereas the lower chambers were maintained with DMEM containing 10\% FBS. Following incubation at $37^{\circ} \mathrm{C}$ for $24 \mathrm{~h}$, the cell suspension in the upper chambers was removed and cells migrated to the lower chambers were stained with $0.1 \%$ crystal violet for $15 \mathrm{~min}$ at room temperature. The migrated cells were counted randomly in at least five fields each time under an inverted microscope.

Statistical analysis. All experiments were performed in triplicate and data are presented as the mean \pm standard deviation. The statistical significance of the differences between groups was assessed using one-way analysis of variance followed by a post hoc Tukey test for multiple comparisons. Statistical analysis was performed using SPSS software version 15.0 (SPSS, Inc., Chicago, IL, USA). P $<0.05$ was considered to indicate a statistically significant difference.

\section{Results}

Amplification of the human full-length ARTS gene in LX-2 cells. To investigate the effect of the overexpression of ARTS on LX-2 cells, the full-length ARTS was amplified. RT-PCR results demonstrated that there was a visible DNA band at $\sim 800 \mathrm{bp}$, which correspond to the target gene ARTS, as determined by $1 \%$ agarose gel electrophoresis (full length ARTS, 846bp; Fig. 2).

Construction and identification of recombinant pEASY-T1-ARTS and pIRES2-EGFP-ARTS plasmids. The recombinant pEASY-T1-ARTS was confirmed by PCR as described in the materials and methods, and the results in Fig. 3A demonstrate that two PCR products were observed in lanes 2 and 3 with sizes that match the expected sizes (223 bp and $99 \mathrm{bp}$, respectively). In the construction of the 


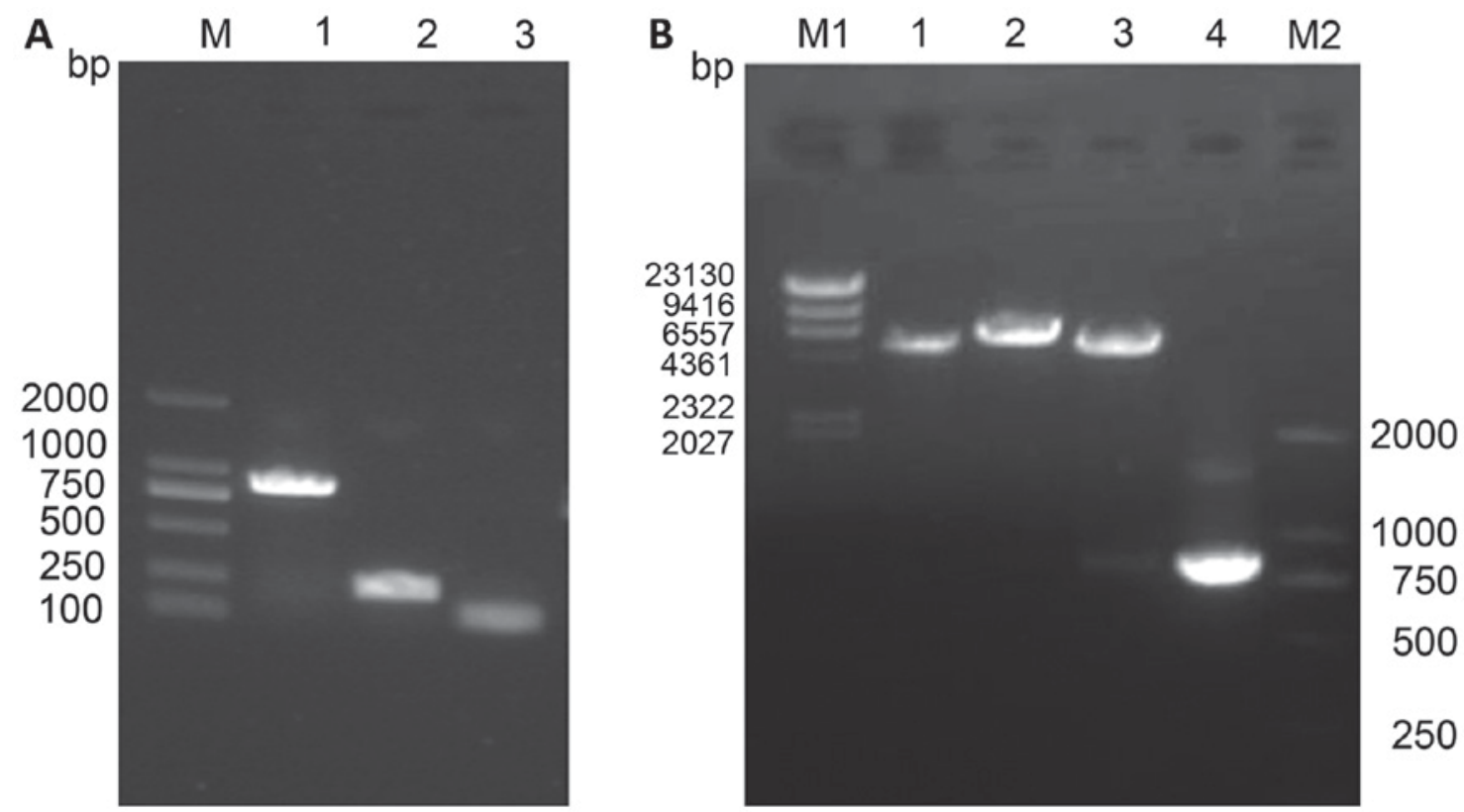

Figure 3. Identification of recombinant plasmids pEASY-T1-ARTS and pIRES2-EGFP-ARTS. (A) Identification of pEASY-T1-ARTS recombinant plasmid. Lane M, DL2000 DNA marker; lane 1, RT-PCR products of ARTS gene; lanes 2 and 3, PCR verified products (223 and 99 bp, respectively). (B) Identification of recombinant plasmid pIRES2-EGFP-ARTS. Lane M1, $\lambda$-HindIII DNA marker; lane 1, recombinant plasmid pIRES2-EGFP-ARTS; lane 2, recombinant plasmid single enzyme digestion; lane 3, recombinant plasmid double enzyme digestion with BamHI and HindIII; lane 4, ARTS RT-PCR products; lane M2, DL2000 DNA marker. ARTS, apoptosis-related protein in the transforming growth factor- $\beta$ signaling pathway; EGFP, enhanced green fluorescent protein; RT-PCR, reverse transcription-polymerase chain reaction.

Blank

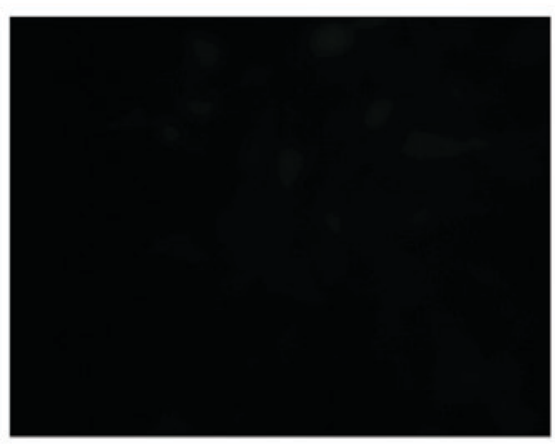

Vector

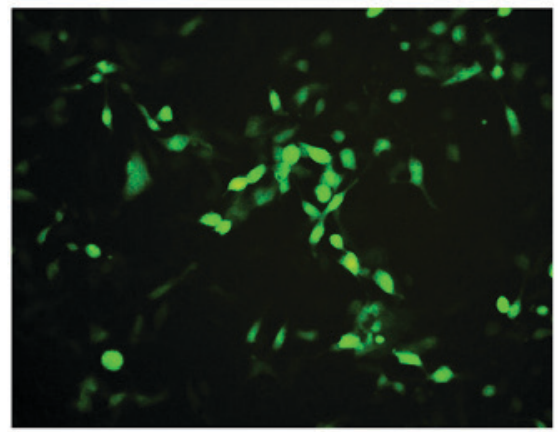

ARTS

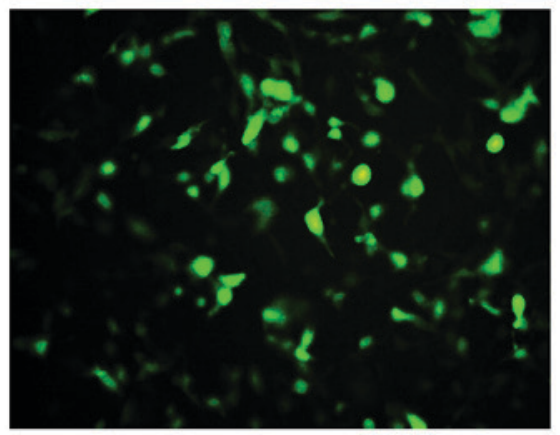

Figure 4. Transfection efficiency of pIRES2-EGFP vector and the recombinant pIRES2-EGFP-ARTS plasmid in LX-2 cells by fluorescence microscopy. Magnification, $x 200$. EGFP, enhanced green fluorescent protein; ARTS, apoptosis-related protein in the transforming growth factor- $\beta$ signaling pathway.

pIRES2-EGFP-ARTS plasmid, double enzyme digestion results demonstrated that a $\sim 800 \mathrm{bp}$ fragment was present in lane 4 (Fig. 3B) The correct recombinant plasmid was successfully constructed for sequencing verification and subsequent experiments.

ARTS induces apoptosis and inhibits activation of LX-2 cells. After $48 \mathrm{~h}$ transfection, EGFP expression was clearly observed in vector and ARTS overexpression groups (Fig. 4). To investigate the effect of ARTS in LX-2 cells, the expression levels of caspase- 3 and $\alpha$-SMA were detected by western blot analysis. As demonstrated in Fig. 5A, the results demonstrated that overexpression of ARTS upregulated the protein levels of cleaved-caspase-3 and downregulated the levels of $\alpha$-SMA, compared with the vector group $(\mathrm{P}<0.05)$. No significant differences were observed between the vector-only group and the blank group ( $\mathrm{P}>0.05)$. TUNEL staining demonstrated that ARTS induced apoptosis in LX-2 cells (Fig. 5B). The apoptotic cells revealed typical morphological changes associated with apoptotic cells, including cell shrinking and rounding. Therefore, these results indicate that ARTS may inhibit the activation of LX-2 cells, based on the effects on $\alpha$-SMA protein levels, and induce their apoptosis.

Overexpression of ARTS has no effect on the proliferation and migration of LX-2 cells. To investigate the effect of ARTS on cell proliferation in LX-2 cells, an MTT assay was performed to evaluate cell survival following transfection. As presented in Fig. 6A, no significant differences were observed among blank, vector and ARTS overexpression groups $(\mathrm{P}>0.05)$. In addition, scratch and Transwell assays were performed to 
A Blank Vector ARTS
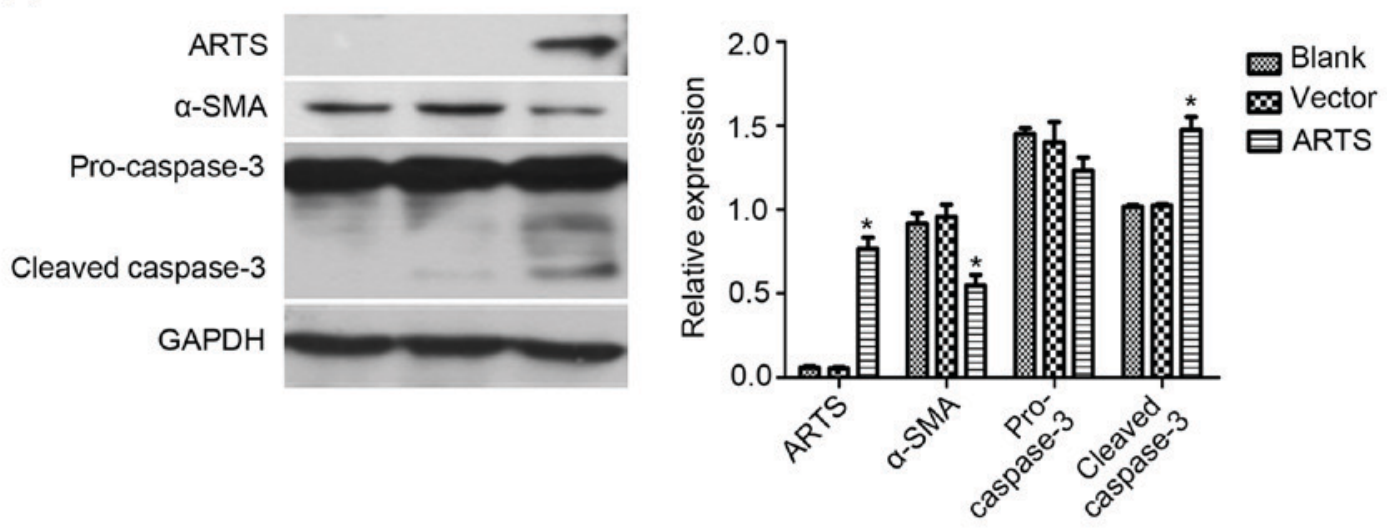

B
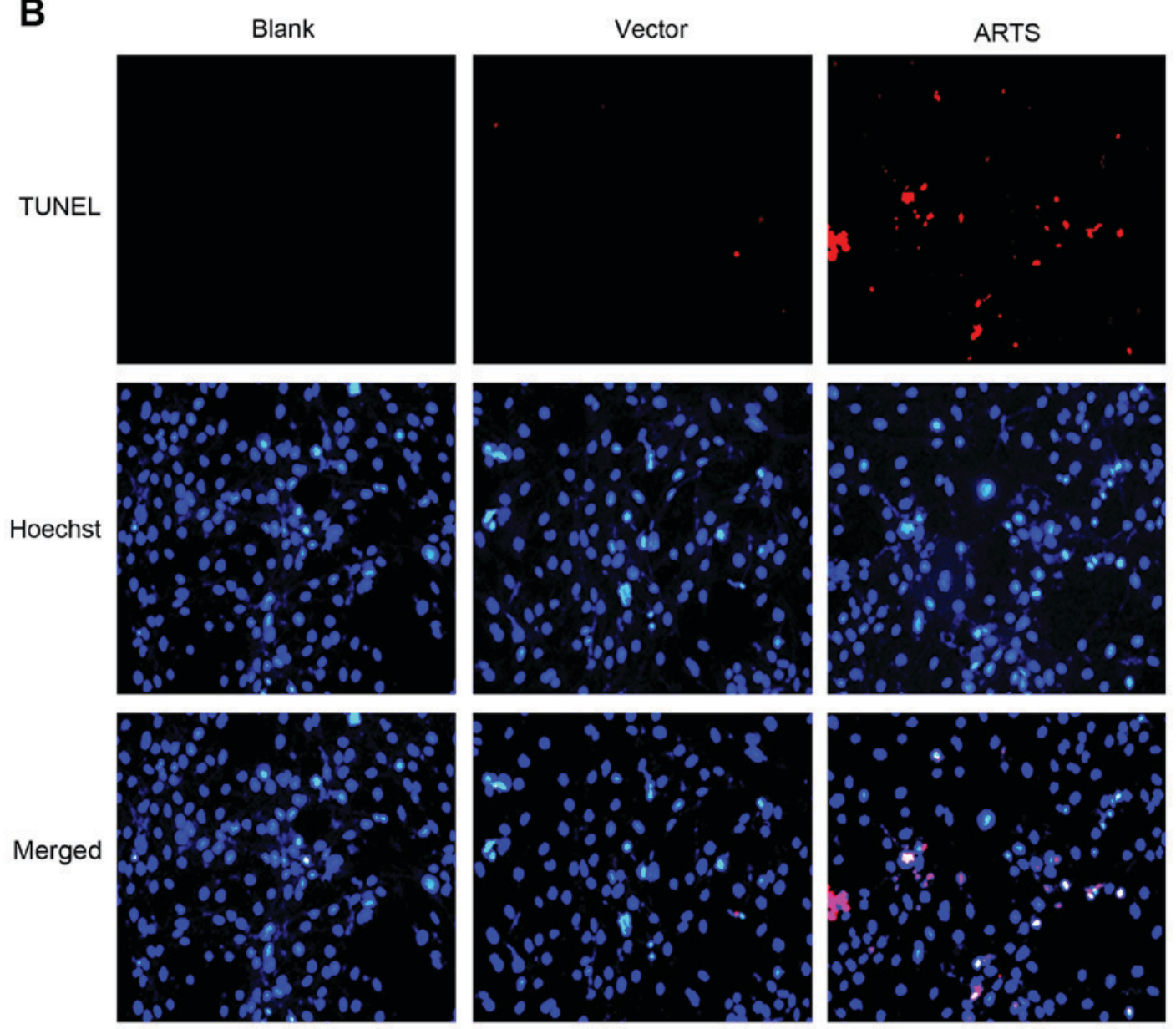

Figure 5. Overexpression of ARTS induced activation and inhibited apoptosis in LX-2 cells. (A) Protein expression of ARTS, $\alpha$-SMA, pro-caspase-3 and cleaved-caspase-3 was detected by western blot analysis. Results were evaluated by densitometric analysis with GAPDH as a loading control. ${ }^{*} \mathrm{P}<0.05$ vs. vector group. (B) Overexpression of ARTS increased apoptosis in LX-2 cells as detected by TUNEL staining. Magnification, $\mathrm{x} 200$. Nuclei were stained blue and apoptotic cells were stained red. ARTS, apoptosis related protein in the transforming growth factor- $\beta$ signaling pathway; $\alpha$-SMA, $\alpha$-smooth muscle actin.

determine the effect of ARTS overexpression on the migration of LX-2 cells. Results presented in Fig. 6B-D demonstrate that overexpression of ARTS had no effect on the migration of LX-2 cells $(\mathrm{P}>0.05)$.

\section{Discussion}

Hepatic fibrosis, which involves the activation of HSCs, may be caused by various factors, including persistent liver injuries, chemical ingestion, virus infection and alcoholism (13). Activated HSCs usually secret ECM and express $\alpha$-SMA, while quiescent HSCs primarily express PPAR- $\gamma$ (14). In recent years, apoptosis, senescence, phenotype reversal and immune clearance have been reported to be involved in the clearance of activated HSCs, and apoptosis of activated HSCs may lead to the remission of hepatic fibrosis $(2,13,15,16)$. Therefore, induction of apoptosis in activated HSCs may be a valid strategy for fibrotic reversion. 
A

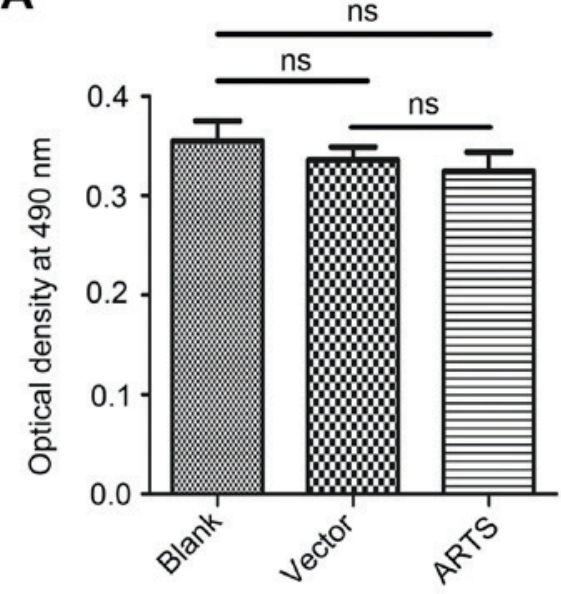

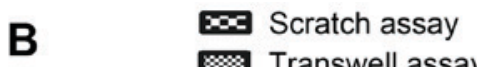

\& Transwell assay

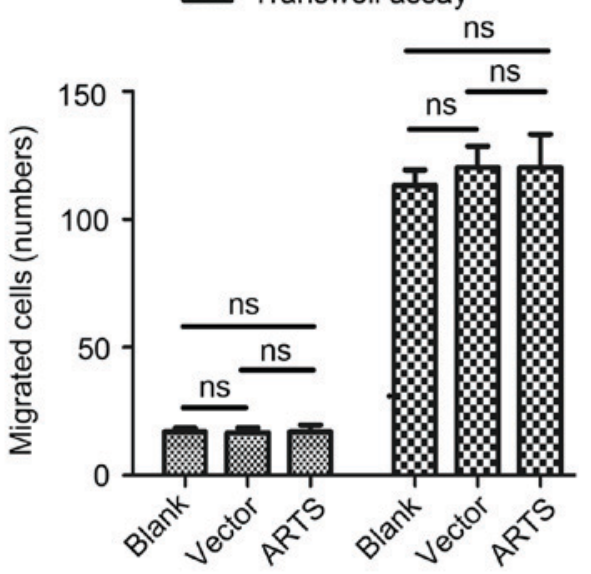

C

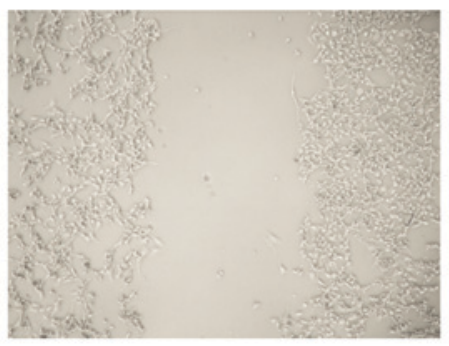

D

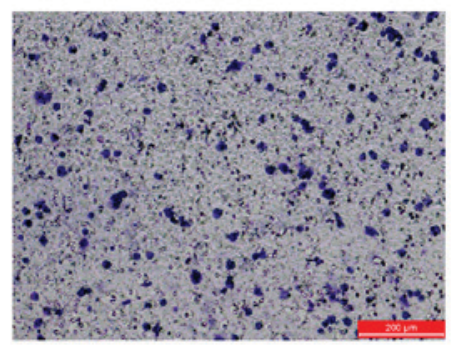

Vector

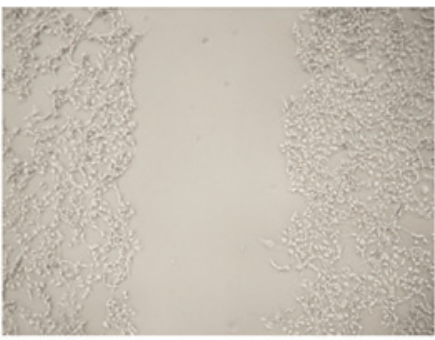

Vector

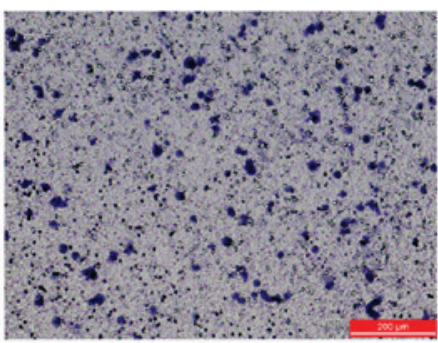

ARTS

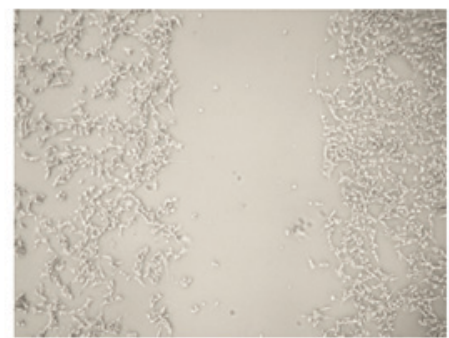

ARTS

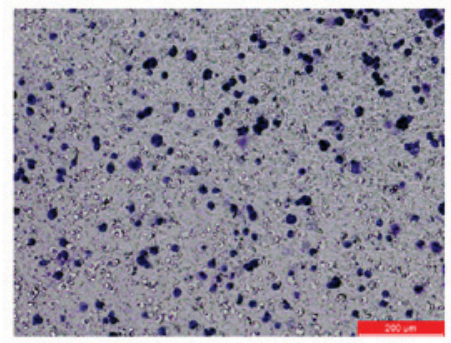

Figure 6. Effect of ARTS overexpression on proliferation and migration in LX-2 cells. (A) MTT assay results demonstrated that overexpression of ARTS had no effect on cell proliferation. (B) Quantified results for scratch and Transwell assays demonstrated that overexpression of ARTS had no effect on cell migration. Representative images for (C) scratch (magnification, $\mathrm{x} 50$ ) and (D) Transwell (magnification, $\mathrm{x} 200$ ) assays. Data are presented as the mean \pm standard deviation of at least three independent experiments. ARTS, apoptosis-related protein in the transforming growth factor- $\beta$ signaling pathway; ns, no significant difference.

ARTS, a splice variant of Sept 4 composed of 846 nucleotides, has been reported to have a role in apoptosis in various cell lines, including A549 and ALLs cells $(8,17)$. Iwaisako et al (12) also reported that Sept4 has a role in hepatic fibrosis, however, the underlying mechanism remains unclear. In addition, Shen et al (18) demonstrated that Sept4_i2 sensitized hepatocellular carcinoma cells to serum starvation-induced apoptosis (18). The results of previous studies also indicated that Sept4_i2 may be involved in Schistosoma japonicum-induced hepatic fibrosis progression, and may regulate apoptosis in HSCs via the PPAR- $\gamma / \mathrm{Akt} / \mathrm{Bcl}-2$ pathway (19-21). Therefore, the present study constructed the eukaryotic recombinant pIRES2-EGFP-ARTS plasmid and determined the effects of ARTS overexpression on LX-2 cells. EGFP is a green fusion protein and has no influence on target gene construction and function (22). EGFP is commonly used as a method for expression modality and gene transfection efficiency detection (23).

In the present study, in order to determine the effect of ARTS on LX-2 cells, the eukaryotic recombinant pIRES2-EGFP-ARTS plasmid was constructed and EGFP was used to observe transfection efficiency and expression. Western blotting and TUNEL staining results demonstrated that overexpression of ARTS induced apoptosis and inhibited activation in LX-2 cells. Therefore, ARTS may be a candidate molecule for treatment against hepatic fibrosis. Our preliminary work may provide the fundamental basis for further research regarding the intracellular signaling mechanisms underlying the effects of ARTS expression in LX-2 cells. 


\section{References}

1. Friedman SL: Molecular regulation of hepatic fibrosis, an integrated cellular response to tissue injury. J Biol Chem 275: 2247-2250, 2000.

2. Elsharkawy AM, Oakley F and Mann DA: The role and regulation hepatic stellate cell apoptosis in reversal of liver fibrosis. Apoptosis 10: 927-939, 2005.

3. Safadi R and Friedman SL: Hepatic fibrosis-role of hepatic stellate cell activation. MedGenMed 4: 27, 2002.

4. Xu L, Hui AY, Albanis E, Arthur MJ, O'Byrne SM, Blaner WS, Mukherjee P, Friedman SL and Eng FJ: Human hepatic stellate cell lines, LX-1 and LX-2: New tools for analysis of hepatic fibrosis. Gut 54: 142-151, 2005.

5. Robert S, Gicquel T, Bodin A, Lagente V and Boichot E: Characterization of the MMP/TIMP imbalance and collagen production induced by IL- $1 \beta$ or TNF- $\alpha$ release from human hepatic stellate cells. PLoS One 11: e0153118, 2016.

6. Liang C, Bu S and Fan X: Suppressive effect of microRNA-29b on hepatic stellate cell activation and its crosstalk with TGF-31/Smad3. Cell Biochem Funct 34: 326-333, 2016.

7. Edison N, Zuri D, Maniv I, Bornstein B, Lev T, Gottfried Y, Kemeny S, Garcia-Fernandez M, Kagan J and Larisch S: The IAP-antagonist ARTS initiates caspase activation upstream of cytochrome C and SMAC/Diablo. Cell Death Differ 19: 356-368, 2012.

8. Gottfried Y, Rotem A, Lotan R, Steller H and Larisch S: The mitochondrial ARTS protein promotes apoptosis through targeting XIAP. EMBO J 23: 1627-1635, 2004.

9. Kemeny S, Dery D, Loboda Y, Rovner M, Lev T, Zuri D, Finberg JP and Larisch S: Parkin promotes degradation of the mitochondrial pro-apoptotic ARTS protein. PLoS One 7: e38837, 2012.

10. Lotan R, Rotem A, Gonen H, Finberg JP, Kemeny S, Steller H, Ciechanover A and Larisch S: Regulation of the proapoptotic ARTS protein by ubiquitin-mediated degradation. J Biol Chem 280: 25802-25810, 2005.

11. Garcia-Fernández M, Kissel H, Brown S, Gorenc T, Schile AJ, Rafii S, Larisch S and Steller H: Sept4/ARTS is required for stem cell apoptosis and tumor suppression. Genes Dev 24: 2282-2293, 2010 .

12. Iwaisako K, Hatano E, Taura K, Nakajima A, Tada M, Seo S, Tamaki N, Sato F, Ikai I, Uemoto S and Kinoshita M: Loss of Sept4 exacerbates liver fibrosis through the dysregulation of hepatic stellate cells. J Hepatol 49: 768-778, 2008.
13. Kong D, Zhang F, Zhang Z, Lu Y and Zheng S: Clearance of activated stellate cells for hepatic fibrosis regression: Molecular basis and translational potential. Biomed Pharmacother 67: 246-250, 2013.

14. Anthony B, Allen JT, Li YS and McManus DP: Hepatic stellate cells and parasite-induced liver fibrosis. Parasit Vectors 3: 60, 2010.

15. Park SJ, Sohn HY, Yoon J and Park SI: Down-regulation of FoxO-dependent c-FLIP expression mediates TRAIL-induced apoptosis in activated hepatic stellate cells. Cell Signal 21: 1495-1503, 2009

16. Wang J, Xu F, Zhu D, Duan Y, Chen J, Sun X, He X, Li P, Sun W and Feng J: Schistosoma japonicum soluble egg antigens facilitate hepatic stellate cell apoptosis by downregulating Akt expression and upregulating p53 and DR5 expression. PLoS Negl Trop Dis 8: e3106, 2014.

17. Elhasid R, Sahar D, Merling A, Zivony Y, Rotem A, Ben-Arush M, Izraeli S, Bercovich D and Larisch S: Mitochondrial pro-apoptotic ARTS protein is lost in the majority of acute lymphoblastic leukemia patients. Oncogene 23: 5468-5475, 2004.

18. Shen S, Liu M, Wu Y, Saiyin H, Liu G and Yu L: Involvement of SEPT4_i1 in hepatocellular carcinoma: SEPT4_i1 regulates susceptibility to apoptosis in hepatocellular carcinoma cells. Mol Biol Rep 39: 4519-4526, 2012.

19. Duan YN, Qian HY, Qin YW, Zhu DD, He XX, Zhou Q, Yang YN, Bao J, Feng JR, Sun W and Chen JL: Dynamics of Sept4 expression in fibrotic livers of mice infected with Schistosoma japonicum. Parasitology 138: 1003-1010, 2011.

20. Zhu D, Wang J, Sun X, Chen J, Duan Y, Pan J, Xu T, Qin Y, He X and Huang C: Septin4_i1 regulates apoptosis in hepatic stellate cells through peroxisome proliferator-activated receptor- $\gamma /$ Akt/B-cell lymphoma 2 pathway. J Histochem Cytochem 63: 163-169, 2015.

21. He X, Bao J, Chen J, Sun X, Wang J, Zhu D, Song K, Peng W, $\mathrm{Xu}$ T and Duan Y: Adenovirus-mediated over-expression of Septin4 ameliorates hepatic fibrosis in mouse livers infected with Schistosoma japonicum. Parasitol Int 67: 487-492, 2015.

22. Cheng A, Zhang Y, Mei H, Fang S, Ji P, Yang J, Yu L and Guo W: Construction of recombinant pEGFP-N1-hPer2 plasmid and its expression in osteosarcoma cells. Oncol Lett 11: 2768-2772, 2016.

23. Petkova MV, Morales-Gonzales S, Relizani K, Gill E, Seifert F, Radke J, Stenzel W, Garcia L, Amthor H and Schuelke M: Characterization of a Dmd (EGFP) reporter mouse as a tool to investigate dystrophin expression. Skelet Muscle 6: 25, 2016. 